\title{
Efficacy and tolerability of DAAs in HCV- monoinfected and HCV/HIV-coinfected patients with psychiatric disorders
}

Nicolò de Gennaro* (D), Lucia Diella, Laura Monno, Gioacchino Angarano, Michele Milella and Annalisa Saracino

\begin{abstract}
Background: Few data are available regarding the use of direct antiviral agents (DAAs) for chronic hepatitis $C$ in psychiatric patients. The aim of the study is to assess safety and outcome of DAAs in patients with psychiatric comorbidities.
\end{abstract}

Methods: This retrospective, observational, single-centre study enrolled patients treated with psychiatric drugs who initiated DAAs between 2015 and 2018. Patients were classified into two groups: A (on anxiolitycs/antidepressant) and B (on antipsychotics). Week-12 sustained virological response (SVR-12) and adverse events (AEs) were evaluated.

Results: One hundred forty-four patients were included (A:101; B:43). Patients were $49.3 \%$ males, mean age 60 years (SD \pm 13.5); 31.9\% cirrhotic; 125 (86.8\%) HCV-monoinfected and 19 (13.2\%) HCV /HIV-coinfected. Twenty patients (13.8\%) required a change of psychiatric therapy before initiation of DAA. Overall, SVR-12 was achieved in $88.2 \%$ of subjects in intention-to-treat(ITT)-analysis. Lower SVR rates were observed in group B vs A (79\% vs 92\%, $p=0.045$ ) and in those changing psychiatric drugs vs others ( $8 \%$ vs $30 \%, p=0.015)$. According to per-protocol (PP)-analysis, SVR-12 was achieved in 93/95 (97.9\%) in group A versus 34/36 (94.4\%) in group B ( $p=0.30)$. At least one AE occurred in 60 patients (41.6\%), including 10 severe AEs, leading to 3 discontinuations. AEs were more frequently reported in group A $(p=0.015)$.

Conclusions: The study confirms effectiveness and safety of DAA-based treatment also in this special population, even if a careful evaluation of history and drug-drug interactions is warranted.

Keywords: HCV, Antiviral treatment, SVR, Psychiatric comorbidity

\section{Background}

Nowadays, it is estimated that 70 million persons are living with hepatitis $\mathrm{C}$ virus (HCV) worldwide, including $1 \%$ of the entire global population according to recent estimates [1]. In addition to the liver-related mortality and morbidity, HCV-infection represents as a systemic disease, as HCV shows a considerable tropism for other tissues and organs [2]. Specifically, HCV-infection has been associated with several

\footnotetext{
* Correspondence: nico84degennaro@gmail.com

Clinic of Infectious Diseases, University of Bari, University Hospital Policlinico, Piazza Giulio Cesare n. 11, 70124 Bari, Italy
}

extrahepatic manifestations among which neuropsychiatric disorders are described in up to half $50 \%$ of chronic HCV-infected patients [3, 4]. Psychiatric symptoms such as cognitive impairment, asthenia, weakness, anxiety and depression have been reported with high frequency in patients with chronic HCVinfection, causing interference with patient ability to perform daily activities and worsening quality of life [5]. This evidence of neurocognitive impairment in some patients with HCV-infection, is not fully attributable to liver dysfunction. Although many researches have suggested a pivotal pathogenetic role of $\mathrm{HCV}$ in

(c) The Author(s). 2020 Open Access This article is licensed under a Creative Commons Attribution 4.0 International License, which permits use, sharing, adaptation, distribution and reproduction in any medium or format, as long as you give appropriate credit to the original author(s) and the source, provide a link to the Creative Commons licence, and indicate if changes were made. The images or other third party material in this article are included in the article's Creative Commons licence, unless indicated otherwise in a credit line to the material. If material is not included in the article's Creative Commons licence and your intended use is not permitted by statutory regulation or exceeds the permitted use, you will need to obtain permission directly from the copyright holder. To view a copy of this licence, visit http://creativecommons.org/licenses/by/4.0/ The Creative Commons Public Domain Dedication waiver (http://creativecommons.org/publicdomain/zero/1.0/) applies to the data made available in this article, unless otherwise stated in a credit line to the data. 
the Central Nervous System (CNS) [6, 7] to explain the high rate of psychiatric comorbidity among these patients, probably the adoption of risk behaviors is the main cause of HCV-infection [8].

Patients with such comorbidity have often been excluded from treatment with Interferon (IFN)-based regimens because of the elevated risk of an exacerbation of psychiatric symptoms [9]. In addition, studies indicated psychiatric comorbidity as a risk factor for nonadherence and not achievement of sustained virological response (SVR) [10]. The recent introduction of IFNfree therapeutic regimens based on direct-acting antiviral agents (DAAs) has revolutionized HCV therapy and viral cure associated with improved quality of life is now a reality in the vast majority of patients. The last therapeutic regimens approved by Food and Drug Administration (FDA) and European Medicines Agency (EMA) are pangenotypic, once-daily, all-oral DAA combinations that have the potential to close the gaps in the current DAA treatment portfolio. Eight-twelve weeks of treatment is now the standard of care and viral eradication can be achieved in $>95 \%$ across different patient populations [11]. However, limited information is currently available regarding the use of DAAs in subjects with psychiatric comorbidity [12-16].

Moreover, the choice of the best correct therapeutic DAA-regimen in these patients is complicated by drugdrug interactions (DDIs) with any psychiatric drug [11].

Therefore, this study seeks to evaluate the efficacy and tolerability of DAA-based antiviral therapy among HCVmonoinfected and HCV/HIV-coinfected patients with psychiatric comorbidity.

\section{Methods}

All consecutive HCV-infected patients ( $\geq 18$ years old) presenting a documented psychiatric comorbidity, with or without HIV infection, who initiated a DAAbased regimen between February 2015 and June 2018 at the Infectious Diseases Clinic, University of Bari, were included in this retrospective, observational study. Patients with a documented psychiatric comorbidity were defined as subjects who had received a previous diagnosis by a psychiatric specialist and for whom a psychiatric drug had been initiated. For purposes of analysis, patients were divided based on their psychiatric treatment into two groups: subjects experienced with anxiolytic and/or antidepressant (group A) and subjects on treatment with antipsychotic (group B).

Demographic, clinical and biochemical data, including type of psychiatric illness and concomitant co-medications at baseline were registered for all subjects.

Potential DDIs were evaluated before the administration of DAAs using a web interaction-checker (available at www.hepdruginteractions.org) and changes in comedications due to DDIs were also recorded. During treatment, all patients routinely underwent monthly monitoring, including clinical and laboratory assessment.

Efficacy assessment (primary endpoint: SVR-12) was based on an intention-to-treat (ITT) analysis, therefore all patients who received at least one dose of anti-HCV medication were included. Moreover, a per protocol (PP)-analysis was performed excluding non compliant and lost to follow-up patients. Non-compliance was evaluated by physicians considering a reported history of incomplete or irregular drug intake.

Safety profile of DAAs (secondary endpoint) was evaluated during each visit in all patients by a dedicated medical equipe, by means of focused questions regarding any sign and/or symptom occurred during treatment. The number of AEs per person was recorded, as well as their type and grade of severity.

\section{DAA-based anti-HCV treatment}

The following regimens were administered based on international recommendations [17]: sofosbuvir (SOF) plus ribavirin (RBV); SOF plus simeprevir (SMV) \pm RBV; SOF plus ledipasvir (LDV) $\pm \mathrm{RBV}$; SOF plus daclatasvir $(\mathrm{DCV}) \pm \mathrm{RBV}$; ombitasvir $(\mathrm{OBV})$, paritaprevir/ritonavir $(\mathrm{PTV} / \mathrm{r}) \pm$ dasabuvir(DSV) $\pm \mathrm{RBV}$, glecaprevir (GLE) plus pibrentasvir (PIB), grazoprevir (GRZ) plus elbasvir (EBR) \pm RBV, SOF plus velpatasvir $(V E L) \pm R B V$. Use of RBV was based on clinical judgement and on the prescribing information document; in particular, an initial dose of $1000 \mathrm{mg}$ or $1200 \mathrm{mg}$ was prescribed according to the patients' weight $(<75$ $\mathrm{kg}$ or $\geq 75 \mathrm{~kg}$, respectively).

\section{HCV-RNA measurement and HCV genotype assessment}

Plasma HCV-RNA levels were measured for all patients at baseline, at the end of treatment (EOT), and 12 weeks after EOT (SVR-12), using the Siemens Real Time PCR assay (Siemens Healthcare Diagnostics, Tarrytown, NY, USA), with a lower limit of detection of $15 \mathrm{IU} / \mathrm{ml}$. HCV genotype and subtype were determined using the Siemens Versant HCV LiPA V2 assay (Siemens, Munich, Germany).

\section{Definitions}

Sustained virological response was defined as an undetectable HCV-RNA level at week 12 after EOT. Virological breakthrough was defined as an undetectable HCV-RNA during treatment followed by a detectable HCV-RNA, despite continued treatment. Relapse was defined as undetectable HCV-RNA at EOT but detectable HCV-RNA during follow-up. 


\section{Severe adverse events (SAEs)}

Severe adverse events were classified according to a recent definition (Common Terminology Criteria for adverse events-CTCAE, 2017).

\section{Statistical analysis}

Descriptive statistics were calculated for demographic, clinical, and laboratory characteristics of cases. Mean and standard deviation (SD) were recorded for normally distributed variables, and the median and interquartile range (IQR) for non-normally distributed variables. The number and percentage were recorded for categorical variables. Differences between groups were analyzed using the Fisher's exact test, or MannWhitney test, as appropriate. A $p$-value of $<0.05$ was considered to indicate significance. Epi Info computer software version 7 was used for statistical analysis.

\section{Ethics}

This research did not require formal approval from the ethics committee according to Italian law, since it was performed in the context of normal clinical routines. However, the study was conducted in accordance with the Declaration of Helsinki and national and institutional standards. All patients provided a written informed consent at the time of first visit at our Centre for the use of their data for research purposes.

\section{Results}

Clinical-demographical features of the study population A total of $1199 \mathrm{HCV}$-infected patients (1081 HCVmonoinfected and $118 \mathrm{HCV} / \mathrm{HIV}$-coinfected) initiated DAAs during the study period. A total of 144/1199 subjects $(12.0 \%)$ presented psychiatric comorbidities and were included in the study, of whom $101(70.1 \%)$ were on anxiolytic/antidepressant therapy (group A) and the remaining $43(29.9 \%)$ were treated with antipsychotic drugs (group B). In Fig. 1 is represented the study diagram.

The clinical characteristics of patients at baseline are summarized in Table 1.

Patients were $49.3 \%$ males with a mean age of 60 years (SD \pm 13.5 ), and $31.9 \%$ of them were cirrhotic. Patients in treatment with antipsychotic drugs were younger (50.6 vs. 64.9 years, $p<0.001)$, mostly males $(67.4 \%$ vs. $41.6 \%, p=0.006)$, the main route of transmission was

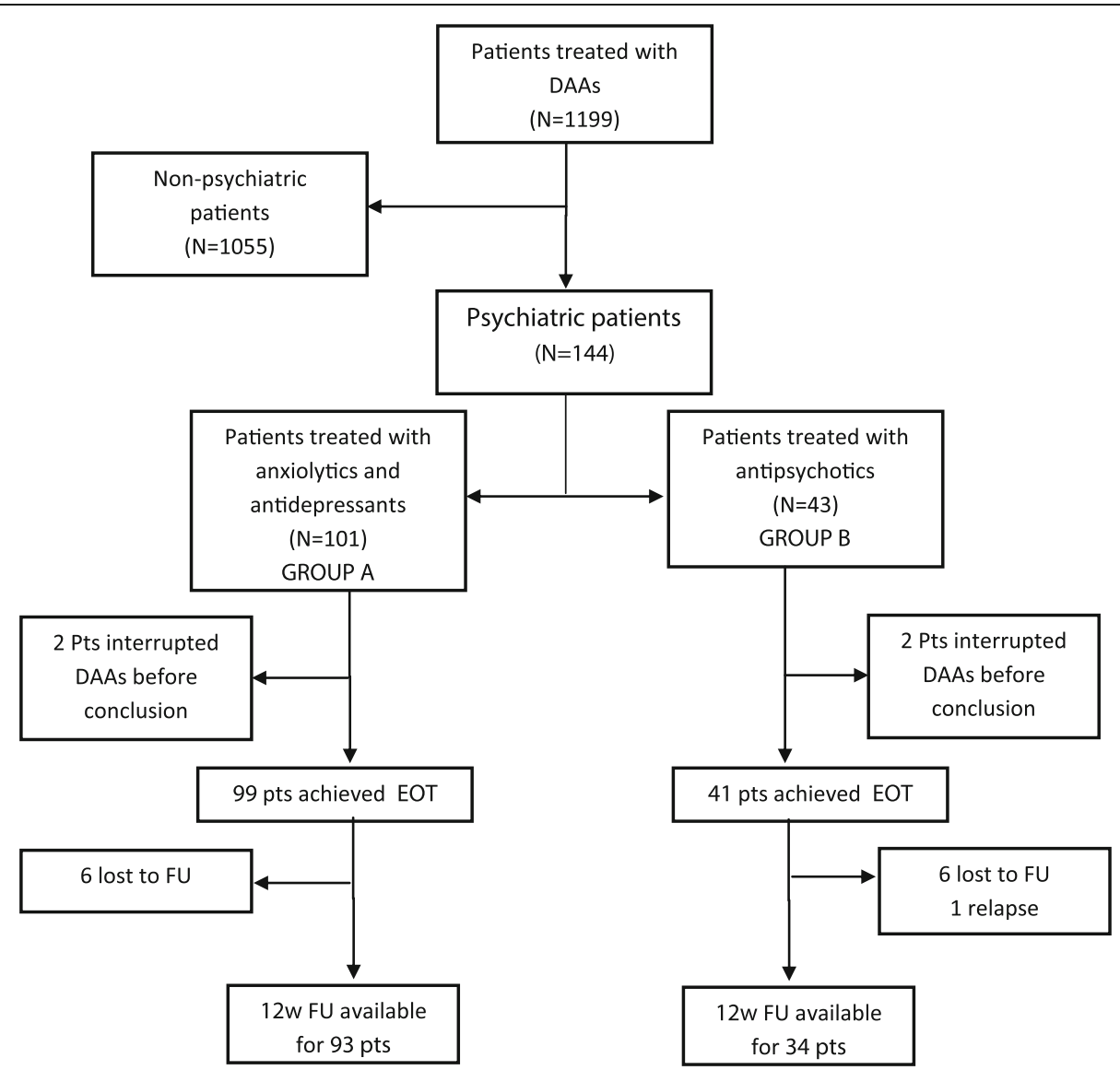

Fig. 1 Flow-chart of pts. enrolled and evaluated from baseline to 12 weeks of follow-up 
Table 1 Baseline features of the study population

\begin{tabular}{|c|c|c|c|c|}
\hline & Patients $(N=144)$ & Group A $(N=101)$ & Group B $(N=43)$ & $p$-value \\
\hline Sex, males. $n(\%)$ & $71(49.3 \%)$ & $42(41.6 \%)$ & $29(67.4 \%)$ & 0.006 \\
\hline Age, years, mean (SD) & $60 \pm 13,5$ & $64.9 \pm 11.7$ & $50.6 \pm 12.1$ & $<0.001$ \\
\hline \multicolumn{5}{|l|}{ Risk Factors $n$ (\%) } \\
\hline IDU & $37(25.7 \%)$ & $17(16.9 \%)$ & $20(46.5 \%)$ & $<0.001$ \\
\hline Heterosexual & $10(7.0 \%)$ & $8(7.9 \%)$ & $2(4.7 \%)$ & 0.720 \\
\hline MSM & $1(0.7 \%)$ & 0 & $1(2.3 \%)$ & 0.290 \\
\hline Nosocomial & $38(26.4 \%)$ & $31(30.6 \%)$ & 7 (16.3\%) & 0.150 \\
\hline Unknown & $58(40.2 \%)$ & $45(44.6 \%)$ & $13(30.2 \%)$ & 0.138 \\
\hline \multicolumn{5}{|l|}{ HCV Genotypes $n$ (\%) } \\
\hline $1 a$ & $32(22.2 \%)$ & $17(16.8 \%)$ & $15(34.9 \%)$ & 0.020 \\
\hline 16 & $51(35.4 \%)$ & $44(43.6 \%)$ & $7(16.3 \%)$ & 0.002 \\
\hline 2 & 37 (25.7\%) & $31(30.7 \%)$ & $6(13.9 \%)$ & 0.038 \\
\hline 3 & $18(12.5 \%)$ & $5(4.9 \%)$ & $13(30.2 \%)$ & $<0.001$ \\
\hline 4 & $6(4.2 \%)$ & $4(4 \%)$ & $2(4.7 \%)$ & 1.000 \\
\hline Duration of $\mathrm{HCV}$ infection, years (range) & $18(10-25.5)$ & $20(11-26)$ & $17(9-23)$ & 0.199 \\
\hline Log 10 HCV-RNA, IU/mL, median (range) & $6.00(5.48-6.52)$ & $5.9(5.6-6.5)$ & $6.2(5.6-6.6)$ & 0.095 \\
\hline Liver stiffness, Kpa, mean (SD) & $10,8 \pm 9,9$ & $11.5 \pm 11.2$ & $9.1 \pm 5.2$ & 0.518 \\
\hline Previous failure, $n(\%)$ & $32(22.2 \%)$ & $29(28.7 \%)$ & $3(7.0 \%)$ & 0.004 \\
\hline INF-based tx & 31 & 28 & 3 & \\
\hline DAAs-based tx & 1 & 1 & 0 & \\
\hline \multicolumn{5}{|l|}{ Fibrosis stage, $n(\%)$} \\
\hline$F 0-F 2$ & $78(54.1 \%)$ & $51(50.5 \%)$ & $27(62.8 \%)$ & 0.203 \\
\hline F3 & $24(16.7 \%)$ & $19(18.8 \%)$ & $5(11.6 \%)$ & 0.338 \\
\hline F4 & $42(29.2 \%)$ & $31(30.7 \%)$ & $11(25.6 \%)$ & 0.689 \\
\hline FIB-4 score median (range) & $2.02(1.40-3.35)$ & $2.2(1.5-3.4)$ & $1.6(1.2-2.9)$ & 0.009 \\
\hline APRI score median (range) & $0.40(0.60-1.22)$ & $0.6(0.4-1.2)$ & $0.7(0.4-1.2)$ & 0.315 \\
\hline MELD score median (range) & $7(6.5-8.5)$ & $7.0(6.0-8.0)$ & $7.0(6.0-7-0)$ & 0.923 \\
\hline \multicolumn{5}{|l|}{ Child-Pugh class $n(\%)$} \\
\hline A & $142(98.6 \%)$ & 100 (99.1\%) & $42(97.7 \%)$ & 0.510 \\
\hline B & $2(1.4 \%)$ & $1(0.99 \%)$ & $1(2.3 \%)$ & 0.510 \\
\hline Cirrhosis, $n$ (\%) & $46(31.9 \%)$ & $34(33.66 \%)$ & $12(27.9 \%)$ & 0.562 \\
\hline Oesophageal varices or portal hypertension, $n(\%)$ & $15(10.4 \%)$ & $11(10.89 \%)$ & $4(9.3 \%)$ & 1.000 \\
\hline \multicolumn{5}{|l|}{ Type of HCV therapy $n$ (\%) } \\
\hline $\mathrm{SOF}+\mathrm{RBV}$ & $7(4.8 \%)$ & $6(5.9 \%)$ & $1(2.3 \%)$ & 0.670 \\
\hline$S O F+S M V \pm R B V$ & $5(3.5 \%)$ & $4(3.9 \%)$ & $1(2.3 \%)$ & 1.000 \\
\hline$S O F+L D V \pm R B V$ & $21(14.6 \%)$ & $15(14.9 \%)$ & $6(13.9 \%)$ & 1.000 \\
\hline$S O F+D C V \pm R B V$ & $13(9.0 \%)$ & $10(10 \%)$ & $3(7 \%)$ & 0.750 \\
\hline $\mathrm{OMB}+\mathrm{PTV} / \mathrm{r}+\mathrm{DAS} \pm \mathrm{RBV}$ & $15(10.4 \%)$ & $15(14.9 \%)$ & 0 & 0.006 \\
\hline $\mathrm{OMB}+\mathrm{PTV} / \mathrm{r}+\mathrm{RBV}$ & $2(1.4 \%)$ & $2(1.9 \%)$ & 0 & 1.000 \\
\hline$S O F+V E L \pm R B V$ & 37 (25.7\%) & $15(14.9 \%)$ & $22(51.2 \%)$ & $<0.001$ \\
\hline $\mathrm{GLE}+\mathrm{PIB}$ & $24(16.7 \%)$ & $17(16.8 \%)$ & $7(16.3 \%)$ & 1.000 \\
\hline $\mathrm{GRZ}+\mathrm{EBR} \pm \mathrm{RBV}$ & $20(13.9 \%)$ & $17(16.8 \%)$ & $3(7 \%)$ & 0.186 \\
\hline \multicolumn{5}{|l|}{ Duration of therapy $n(\%)$} \\
\hline $8 w$ & $20(13.9 \%)$ & $15(14.9 \%)$ & $5(11.6 \%)$ & 0.794 \\
\hline
\end{tabular}


Table 1 Baseline features of the study population (Continued)

\begin{tabular}{|c|c|c|c|c|}
\hline & Patients $(N=144)$ & Group A $(N=101)$ & Group B $(N=43)$ & $p$-value \\
\hline $12 w$ & $101(70.1 \%)$ & $67(66.3 \%)$ & $34(79.1 \%)$ & 0.164 \\
\hline $16 w$ & $6(4.2 \%)$ & $4(3.9 \%)$ & $2(4.65 \%)$ & 1.000 \\
\hline $24 w$ & $17(11.8 \%)$ & $15(14.9 \%)$ & $2(4.65 \%)$ & 0.097 \\
\hline Addition of RBV $n$ (\%) & $33(22.9 \%)$ & $24(23.74 \%)$ & $9(20.9 \%)$ & 0.829 \\
\hline ALT, UI/L, median (range) & $50(30-80)$ & $47(26.5-78.5)$ & $58(43-93)$ & 0.029 \\
\hline Total bilirubin, mg/dL, median (range) & $0.62(0.45-0.85)$ & $0.65(0.47-0.82)$ & $0.60(0.40-0.87)$ & 0.323 \\
\hline Serum creatinine, $\mathrm{mg} / \mathrm{dL}$, median (range) & $0.78(0.61-0.93)$ & $0.78(0.60-0.95)$ & $0.78(0.62-0.90)$ & 0.795 \\
\hline Platelets $\left(\times 10^{\wedge} 9 / \mathrm{L}\right)$, median (range) & $183.5(140.5-217.5)$ & $184(145-219)$ & $182(137-214)$ & 0.936 \\
\hline Albumin, mg/dL, median (range) & $4.0(3.7-4.2)$ & $4.0(4.7-4.2)$ & $4.0(3.8-4.1)$ & 0.976 \\
\hline Body mass index, median (range) & $25.3(23.3-28.7)$ & $25.5(23.8-29.1)$ & $25.1(22.4-27.4)$ & 0.936 \\
\hline Patients with at least 1 other comorbidity, $\mathrm{n}(\%)$ & $101(70.10 \%)$ & 79 (78.2\%) & $22(51.2 \%)$ & 0.002 \\
\hline Diabetes, $\boldsymbol{n}(\%)$ & $20(13.9 \%)$ & $16(15.8 \%)$ & $4(9.3 \%)$ & 0.431 \\
\hline Hypertension, $n$ (\%) & $61(42.4 \%)$ & $53(52.5 \%)$ & $8(18.6 \%)$ & $<0.001$ \\
\hline HBsAg positive, $n(\%)$ & $2(1.4 \%)$ & 0 & $2(4.7 \%)$ & 0.087 \\
\hline HIV infected, $n(\%)$ & $19(13.2 \%)$ & $9(8.9 \%)$ & $10(23.3 \%)$ & 0.030 \\
\hline$N$ aviremic pts on $A R T, n(\%)$ & $19(100 \%)$ & $9(100 \%)$ & $10(100 \%)$ & 1.000 \\
\hline $\mathrm{CD}^{+}$(cells/ $\mu$ l) median (range) & $85(42-171)$ & $98(50-171)$ & $83.5(42-186.5)$ & 0.903 \\
\hline CDC-C Stage $n(\%)$ & $7(4.8 \%)$ & $4(3.9 \%)$ & $3(7 \%)$ & 0.650 \\
\hline
\end{tabular}

Legend: IDU Injecting drug user, MSM Man who have sex with man, INF Interferon, DAA Direct antiviral agent, SOF Sofosbuvir, RBV Ribavirin, SMV Simeprevir, LDV Ledipasvir, DCV Daclatasvir, OMB Ombitasvir, PTV/r Paripatrevir/ritonavir, DSV Dasabuvir, VEL Velpatasvir, GLE Glecaprevir, PIB Pibrentasvir, GRZ Grazoprevir, EBR Elbasvir, ALT Alanine aminotransferase, ART Antiretroviral therapy

intravenous drug use $(46.5 \%$ vs. $16.9 \%, p<0.001)$ and were more frequently infected with $\mathrm{HCV}$ genotype 1a (34.9\% vs. $16.8 \%, p=0.020)$, and genotype 3 (30.2\% vs. $4.9 \%, p<0.001)$.

Most patients (70.1\% of the study population) had at least one comorbidity (other than psychiatric). Group A individuals were more likely to have at least one additional comorbidity before starting DAA-treatment (78.2\% vs. $51.2 \%, p=0.002)$. Arterial hypertension was the most common reported comorbidity (42.4\%), mainly diagnosed in patients belonging to group A $(52.5 \%$ vs. $18.6 \%, p<0.001)$.

Failure to a previous anti-HCV treatment was reported in 32 patients $(22.2 \%)$, the majority among patients in treatment with anxiolitycs and antidepressant $(28.7 \%$ vs. $7.0 \%, p=0.004)$. Among the HCV treatmentexperienced subjects, one had experienced failure with a previous DAA-based regimen.

All patients had compensated liver disease according to the Child-Pugh classification. Group A subjects showed a higher FIB- 4 score (2.2 vs. $1.6, p=0.009)$. No differences in liver stiffness and fibrosis stage distribution, APRI/MELD score, and HCV-RNA at baseline were observed between the two groups.

Furthermore, 125 (86.8\%) were HCV-monoinfected and 19 (13.2\%) were HCV/HIV-coinfected. Coinfected patients were mostly represented among subjects in treatment with antipsychotic drugs (23.3\% vs. $8.9 \%, p=$ 0.030 ). Two patients had HBsAg positivity, both in Group B.

DAA-regimens were chosen by treating clinicians based on clinical criteria and viral genotype. The most frequently prescribed DAA-regimen was SOF $+\mathrm{VEL} \pm$ RBV (25.7\% overall), especially in subjects in treatment with antipsychotic $(51.2 \%$ vs. $14.9 \%, p<0.001)$. The regimen including $\mathrm{OMB}+\mathrm{PTV} / \mathrm{r} \pm \mathrm{DAS} \pm \mathrm{RBV}$ was never prescribed in group B patients $(p=0.006)$.

In Table 2 the characteristics of the psychiatric disorder are detailed for both groups. Subjects belonging to group B, which included a higher percentage of former drug users, were mostly on substitution treatment with opioids at the time of inclusion in the study. The psychiatric illness' duration was longer in group B $(p=0.011)$. No statistically significant differences between the two groups were found regarding the referred alcohol consumption.

\section{Change of psychiatric and antiretroviral treatment before DAAs-initiation}

Among the entire study population, a total of 20 patients (13.8\%) required a modification of psychiatric therapy based on DDIs in according to the psychiatrist' judgment before the beginning of the DAA-regimen: a dose adjustment of psychiatric drugs was observed in two patients 
Table 2 Clinical characteristics concerning psychiatric disorders at baseline

\begin{tabular}{|c|c|c|c|c|}
\hline & Patients $(N=144)$ & Group A & Group B & $\boldsymbol{p}$-value \\
\hline Types of psychiatric illnesses, $n$ (\%) & & $101(70.1)$ & $43(29.9 \%)$ & \\
\hline Anxiety disorders ${ }^{a}$ & & $48(33.3 \%)$ & & \\
\hline Mood disorders ${ }^{b}$ & & $53(36.8 \%)$ & & \\
\hline Psychotic disorder & & & $43(29.9 \%)$ & \\
\hline Duration psychiatric illness, years (range) & $9(6-13)$ & $8(6.5-12.5)$ & $12(8-15.5)$ & 0.011 \\
\hline Suicide attempted, $\boldsymbol{n}(\%)$ & $5(3.5 \%)$ & $2(2 \%)$ & $3(7 \%)$ & 0.157 \\
\hline Psychiatric treatment modification before DAA treatment, $n(\%)$ & $20(13.9 \%)$ & $11(10.9 \%)$ & $9(21 \%)$ & 0.121 \\
\hline Opioid substitution treatment, $n$ (\%) & $12(8.3 \%)$ & $2(2 \%)$ & $10(23.3 \%)$ & $<0.001$ \\
\hline Methadone & $11(7.6 \%)$ & $1(1 \%)$ & $10(23.3 \%)$ & $<0.001$ \\
\hline Buprenorphine & $1(0.7 \%)$ & $1(1 \%)$ & 0 & 1.000 \\
\hline Referred alcohol consumption, $n(\%)$ & $5(3.5 \%)$ & $4(4 \%)$ & $1(2.3 \%)$ & 1.000 \\
\hline
\end{tabular}

Legend: ${ }^{\text {a }}$-social phobia-posttraumatic stress disorder-panic disorder-generalized anxiety disorder;

b-current major depressive episode-current manic episode-current hypomanic episode;

whereas in the remaining 18 patients (10 in group A $9.9 \%$ and 8 in group $\mathrm{B}, 18.6 \%, p=0.172)$ a complete therapeutic change was adopted. In seven cases $(7 / 20$, $35 \%)$ a therapeutic change was mandatory due to drugdrug interactions.

Regimens more likely to require a change of psychiatric drugs before starting DAA-treatment were SOF + $\mathrm{DCV} \pm \mathrm{RBV}$ (3/13 patients, 23.1\%); GRZ + EBR $\pm \mathrm{RBV}$ (4/20, 20\%), SOF + VEL \pm RBV $(7 / 37,18.9 \%)$, and GLE + PIB (3/24, 12.5\%).

Among the $19 \mathrm{HIV} / \mathrm{HCV}$-coinfected individuals, all patients were on ART at the initiation of DAAtreatment and were aviremic. A total of 6/19 patients (31.6\%) required a change of ART because of DDIs, of whom four patients from group A and two from group B $(p=0.349)$. Before the ART change, 5/6 (83.3\%) patients were in treatment with protease inhibitors: one with atazanavir unboosted, two with lopinavir/ritonavir, two with darunavir/ritonavir. Of the 6 patients who changed ART, only one returned to his previous ART regimen after the end of DAA-treatment. No virological failure for HIV was observed.

\section{Outcome}

Among the 144 patients enrolled, 140/144 (97.2\%) completed the prescribed DAA-regimen accomplishing EOT, while four patients prematurely interrupted their therapy: in one case due to imprisonment, and in the remaining three cases due to the occurrence of AEs (see the following paragraph).

Overall, according to an ITT-analysis, SVR-12 was achieved in $127 / 144$ subjects $(88.2 \%)$ of the study population, 93/101 (92.1\%) in group A versus 34/43 (79.1\%) in group $\mathrm{B}$, respectively $(p=0.045)$. Clinical characteristics of psychiatric patients stratified by achieving SVR-12 are detailed in Table 3.
Among the patients achieving EOT but not SVR-12, twelve were lost to follow-up (6 from group A and 6 from group B), and only one (belonging to group B) had a relapse. This patient was a non-cirrhotic subject infected with HCV genotype 3 , who had received the $\mathrm{SOF}+\mathrm{DCV}+\mathrm{RBV}$ combination for 12 weeks; he had resistance testing performed at failure which demonstrated a resistance pattern against NS5A (Y93H).

A lower SVR rate (79\%), was observed in psychotic patients (group B) compared to group A (92\%) which was mostly due to a higher prevalence of patients lost to follow-up $(6 / 43,14 \%$ vs. $6 / 101,6 \%$ respectively, $p=$ 0.183).

Patients who did not achieve SVR-12 were mostly males $(76.5 \%$ vs $45.7 \%$ in responders patient, $p=0.020)$, younger ( 51.6 vs $61.8, p=0.038$ ), more frequently intravenous drug users $(52.9 \%$ vs $22 \%, p=0.014)$ and presented a higher rate of failure to a previous anti-HCV treatment $(47.0 \%$ vs $18.9 \%, p=0.024)$. The proportion of patients not achieving SVR-12 was higher among patients who underwent a change of the psychiatric regimen before anti-HCV treatment (6/20 patients, 30\%) compared to those who maintained the same psychiatric therapy $(11 / 124,8.8 \%)(p=0.015)$. On the contrary, no differences were observed according to ART change before anti-HCV treatment (no patient failed among those changing ART before baseline while only two failures were evidenced among the remaining subjects).

Excluding lost to follow-up and non compliant patients, according to PP-analysis, SVR-12 was achieved in 93/95 (97.9\%) in group A versus 34/36 (94.4\%) in group $\mathrm{B}(p=0.30)$.

\section{Safety profile of DAAs}

The safety profile was evaluated for the 144 enrolled subjects, and is described in Table 4. Treatment 
Table 3 Clinical characteristics of psychiatric patients stratified by achieving SVR-12

\begin{tabular}{|c|c|c|c|}
\hline & SVR-12 $(N=127)$ & No SVR-12 $(N=17)$ & $p$-value \\
\hline Sex, males. $n(\%)$ & $58(45.7 \%)$ & $13(76.5 \%)$ & 0.020 \\
\hline Age, years, mean (SD) & $61.8 \pm 13.3$ & $51.6 \pm 11.7$ & 0.038 \\
\hline \multicolumn{4}{|l|}{ Risk Factors $n$ (\%) } \\
\hline IDU & $28(22.0 \%)$ & $9(52.9 \%)$ & 0.014 \\
\hline Heterosexual & $10(7.9 \%)$ & 0 & 0.607 \\
\hline MSM & $1(0.8 \%)$ & 0 & 1.000 \\
\hline Nosocomial & $36(28.3 \%)$ & $2(11.8 \%)$ & 0.239 \\
\hline Unknown & $52(41.0 \%)$ & $6(35.3 \%)$ & 0.794 \\
\hline \multicolumn{4}{|l|}{ HCV Genotypes $n(\%)$} \\
\hline $1 a$ & $27(21.3 \%)$ & $5(29.4 \%)$ & 0.534 \\
\hline 16 & $45(35.4 \%)$ & $6(35.3 \%)$ & 1.000 \\
\hline 2 & $35(27.6 \%)$ & $2(11.8 \%)$ & 0.238 \\
\hline 3 & $15(11.8 \%)$ & $3(17.6 \%)$ & 0.448 \\
\hline 4 & $5(3.9 \%)$ & $1(5.9 \%)$ & 0.536 \\
\hline Log 10 HCV-RNA, IU/mL, median (range) & $6.00(5.5-6.5)$ & $6.1(5.5-6.7)$ & 0.841 \\
\hline Liver stiffness, Kpa, mean (SD) & $11.1 \pm 10.4$ & $8.9 \pm 3.4$ & \\
\hline Previous failure, $n(\%)$ & $24(18.9 \%)$ & $8(47.0 \%)$ & 0.024 \\
\hline INF-based tx & 23 & 8 & \\
\hline DAAs-based tx & 1 & 0 & \\
\hline FIB-4 score median (range) & $2.0(1.4-3.3)$ & $1.5(1.3-2.1)$ & 0.226 \\
\hline APRI score median (range) & $0.6(0.4-1.2)$ & $0.6(0.5-1.2)$ & 0.936 \\
\hline MELD score median (range) & $7.0(6.0-8.0)$ & $7.0(6.0-7.0)$ & 0.794 \\
\hline Cirrhosis, $n$ (\%) & $40(31.5 \%)$ & $6(35.3 \%)$ & 0.785 \\
\hline Oesophageal varices or portal hypertension, $n(\%)$ & $13(10.2 \%)$ & $2(11.8 \%)$ & 0.691 \\
\hline \multicolumn{4}{|l|}{ Type of HCV therapy $n(\%)$} \\
\hline $\mathrm{SOF}+\mathrm{RBV}$ & $7(5.5 \%)$ & 0 & 0.594 \\
\hline$S O F+S M V \pm R B V$ & $5(3.9 \%)$ & 0 & 1.000 \\
\hline$S O F+L D V \pm R B V$ & $18(14.2 \%)$ & $3(17.6 \%)$ & 0.715 \\
\hline$S O F+D C V \pm R B V$ & $11(8.7 \%)$ & $2(11.8 \%)$ & 0.652 \\
\hline $\mathrm{OMB}+\mathrm{PTV} / \mathrm{r}+\mathrm{DAS} \pm \mathrm{RBV}$ & $13(10.2 \%)$ & $2(11.8 \%)$ & 0.691 \\
\hline $\mathrm{OMB}+\mathrm{PTV} / \mathrm{r}+\mathrm{RBV}$ & $1(0.8 \%)$ & $1(5.9 \%)$ & 0.222 \\
\hline$S O F+V E L \pm R B V$ & $30(23.6 \%)$ & $7(41.2 \%)$ & 0.141 \\
\hline $\mathrm{GLE}+\mathrm{PIB}$ & $24(18.9 \%)$ & 0 & 0.076 \\
\hline$G R Z+E B R \pm R B V$ & $18(14.2 \%)$ & $2(11.8 \%)$ & 1.000 \\
\hline Addition of RBV $n$ (\%) & $27(21.2 \%)$ & $6(35.3 \%)$ & 0.222 \\
\hline ALT, UI/L, median (range) & $50(28.5-77.5)$ & $70(47-125)$ & 0.031 \\
\hline Total bilirubin, mg/dL, median (range) & $0.62(0.44-0.83)$ & $0.60(0.40-0.80)$ & 0.561 \\
\hline Serum creatinine, mg/dL, median (range) & $0.79(0.61-0.95)$ & $0.78(0.63-0.80)$ & 0.928 \\
\hline Platelets $\left(\times 10^{\wedge} 9 / \mathrm{L}\right)$, median (range) & $182(139-219)$ & $185(157-210)$ & 0.920 \\
\hline
\end{tabular}


Table 3 Clinical characteristics of psychiatric patients stratified by achieving SVR-12 (Continued)

\begin{tabular}{llll}
\hline & SVR-12 (N=127) & No SVR-12 (N=17) & $\boldsymbol{p}$-value \\
\hline Patients with at least $\mathbf{1}$ other comorbidity, $\mathbf{n}(\%)$ & $94(74 \%)$ & $7(41.2 \%)$ & $\mathbf{0 . 0 0 9}$ \\
Diabetes, $\boldsymbol{n}(\%)$ & $18(14.2 \%)$ & $2(11.8 \%)$ & 1.000 \\
Hypertension, $\boldsymbol{n}(\%)$ & $57(44.9 \%)$ & $4(23.5 \%)$ & 0.119 \\
HIV infected, $\boldsymbol{n}(\%)$ & $17(13.4 \%)$ & $2(11.8 \%)$ & 1.000 \\
\hline
\end{tabular}

Legend: IDU Injecting drug user, MSM Man who have sex with man, INF Interferon, DAA Direct antiviral agent, SOF Sofosbuvir, RBV Ribavirin, SMV Simeprevir, LDV Ledipasvir, DCV Daclatasvir, OMB Ombitasvir, PTV/r Paripatrevir/ritonavir, DSV Dasabuvir, VEL Velpatasvir, GLE Glecaprevir, PIB Pibrentasvir, GRZ Grazoprevir, EBR Elbasvir, ALT Alanine aminotransferase, ART Antiretroviral therapy

discontinuation was observed in 4 (2.7\%) individuals of the study population and were due to: seizures incoming, syncopal episode, severe headache; the fourth patient was detained and interrupted the treatment. A total of $60 \mathrm{pa}-$ tients (41.6\%) experienced at least one AE. Most AEs in course of DAAs occurred in patients in treatment with anxiolytics and antidepressant $(48.5 \%$ vs. $25.6 \%, p=0.015)$. The most common AEs were represented by neurological symptoms (18.0\%), skin reactions (7.6\%), and gastrointestinal disorders (7.6\%). Patients receiving RBV more frequently showed AEs during treatment in comparison with subjects treated with RBV-sparing regimens. Erythropoietin use and a RBV dose reduction were reported in 2 $(1.3 \%)$ and $4(2.7 \%)$ patients, respectively. No statistically significant differences in terms of occurrence of AEs were observed between the two groups. Psychiatric symptoms such as anxiety episodes and mood disorders were reported only among group A patients.

Severe AEs were generally uncommon (6.9\%). The most common SAE was severe anaemia in 3 patients (2.1\%). Three hospitalizations (not leading to treatment discontinuation) were reported due to: angina episode, hyperammonaemic encephalopathy and hematemesis. No death was reported.

\section{Discussion}

The treatment of chronic hepatitis $C$ virus infection has been revolutionized thanks to the recent development of the new oral direct-acting antiviral agents. DAAs increase the likelihood of cure with a shorter duration of

Table 4 Safety profile of DAAs regimens

\begin{tabular}{|c|c|c|c|c|}
\hline & Patients $(N=144)$ & Group A $(N=101)$ & Group B $(N=43)$ & $p$-value \\
\hline At least 1 adverse event, $n(\%)$ & $60(41,6 \%)$ & $49(48.5 \%)$ & $11(25.6 \%)$ & 0.015 \\
\hline$>2$ adverse events, $n(\%)$ & $7(4.8 \%)$ & $6(5.9 \%)$ & $1(2.3 \%)$ & 0.674 \\
\hline Severe adverse events, $\boldsymbol{n}(\%)$ & $10(6.9 \%)$ & $8(7.9 \%)$ & $2(4.6 \%)$ & 0.723 \\
\hline Adverse events leading to discontinuation, $n(\%)$ & $3(2.1 \%)$ & $2(1.9 \%)$ & $1(2.3 \%)$ & 1.000 \\
\hline Skin reactions ${ }^{\mathrm{a}}, n(\%)$ & $11(7.6 \%)$ & $9(8.9 \%)$ & $2(4.6 \%)$ & 0.506 \\
\hline Anemia, $n(\%)$ & $7(4.8 \%)$ & $5(4.9 \%)$ & $2(4.6 \%)$ & 1.000 \\
\hline Requiring erythropoietin & $2(1.3 \%)$ & $2(1.9 \%)$ & 0 & \\
\hline Requiring RBV dose adjustment & $4(2.7 \%)$ & $2(1.9 \%)$ & $2(4.6 \%)$ & \\
\hline Requiring hospitalization & $1(0.7 \%)$ & $1(0.9 \%)$ & 0 & \\
\hline Gastrointestinal toxicity ${ }^{b}, n(\%)$ & $11(7.6 \%)$ & $9(8.9 \%)$ & $2(4.6 \%)$ & 0.506 \\
\hline Cardiac disorders ${ }^{c}, n(\%)$ & $6(4.1 \%)$ & $5(4.9 \%)$ & $1(2.3 \%)$ & 0.669 \\
\hline Neurological symtomps, $n$ (\%) & $26(18.0 \%)$ & $20(19.8 \%)$ & $6(13.9 \%)$ & 0.483 \\
\hline Asthenia & $15(10.4 \%)$ & $12(11.8 \%)$ & $3(6.9 \%)$ & 0.553 \\
\hline Headache & $10(6.9 \%)$ & $9(8.9 \%)$ & $1(2.3 \%)$ & 0.281 \\
\hline Insomnia & $8(5.5 \%)$ & $6(5.9 \%)$ & $2(4.6 \%)$ & 1.000 \\
\hline Amnesia & $1(0.7 \%)$ & $1(0.9 \%)$ & 0 & 1.000 \\
\hline Seizures & $1(0.7 \%)$ & 0 & $1(2.4 \%)$ & 0.298 \\
\hline Psychiatric symtomps, $n$ (\%) & $8(5.5 \%)$ & $8(7.9 \%)$ & 0 & 0.105 \\
\hline Anxiety & $1(0.7 \%)$ & $1(0.9 \%)$ & 0 & 1.000 \\
\hline Mood disorders & $7(4.8 \%)$ & $7(6.9 \%)$ & 0 & 0.103 \\
\hline
\end{tabular}

Legend: ${ }^{\mathrm{a}}$-rash, pruritus, photosensitivity;

b- diarrhea/constipation, dyspepsia, nausea;

c-hyper/hypotension, arrhythmias 
treatment and a better safety profile compared to previously used IFN-based regimens.

Despite these overwhelming advances, challenges remain in eliminating $\mathrm{HCV}$ in some patient subgroups, such as subjects with decompensated cirrhosis, severe kidney disease, and in the elderly [18]. In addition, among these vulnerable populations, also patients who have psychiatric disorders should be taken into account, for whom IFN-treatment was eluded in previous years or discontinued for severe long-term and incapacitating neuropsychiatric side effects [9]. A certain reluctance of physicians to treat this "special group" can be attributed to concerns about poor treatment adherence or pessimism regarding HCV-treatment tolerability and/or effectiveness even in the DAA era [19]. For all the above mentioned reasons, nowadays these patients are still lacking or delaying access to treatment and cure.

The coexistence of HCV-infection with cognitive disorders is well known, based on studies demonstrating the entrance of $\mathrm{HCV}$ in $\mathrm{CNS}$ as well as the higher exposure to $\mathrm{HCV}$-infection in patients with psychiatric comorbidities due to more frequent risk behaviors compared to the general population [20]. However, if depression and/or anxiety have been reported in about a third of $\mathrm{HCV}$-infected patients according to different studies [21-23], the prevalence of psychotic disorders (such as schizophrenia, delirious disorder) among HCV infected subjects is not well established, but is estimated about $4 \%$ [24]. In our analysis, the overall proportion of $\mathrm{HCV}$-infected patients with psychiatric comorbidity who underwent antiviral therapy was lower than in the above mentioned studies (about 12\%), even if restricted inclusion criteria were used, as only patients under a psychiatric treatment were considered eligible.

Our study confirms findings showing that HCVpatients with psychiatric co-morbidity can be treated with DAAs with high efficacy. The rate of SVR-12 obtained in our study (88.2\% in ITT-analysis) was consistent with the recent results highlighted from Sundberg et al [25] in whose study 17 patients, despite severe psychiatric morbidity, successfully completed DAAtreatment with a comparable SVR (88\%), without worsening psychiatric symptoms. Only few data are available on SVR rates in patients with psychiatric comorbidites $[13,16]$. These SVR rates appear only slightly lower compared to those currently reported in the general HCV-infected population, also in our real life experience [26], while it seems to be lower in intravenous drug addicted [14]; We tried to further investigate reasons underlying this gap and for these purposes the population was divided into two groups, based on clinical and therapeutic resemblance, including patients treated with anxiolytics and/or antidepressant in group A, and patients treated with antipsychotics in group $B$ and we find that psychotic patients presented lower SVR rates. It should be beforehand underlined that the two groups were not homogeneous. A first imbalance pertains to the different number of patients in the two groups, with more than twice subjects in group A. A higher percentage of former IDUs was observed in the group of psychotic patients and the population of $\mathrm{HCV} / \mathrm{HIV}$-coinfected patients was more represented. As a consequence, differences in the genotype distribution were evidenced: in fact, as expected, genotype $1 \mathrm{a}$ and 3 were found widely in group B patients. In contrast, patients in treatment with anxiolytics/antidepressants were significantly older than those in group $\mathrm{B}$, and consequently presented a higher FIB-4 score at baseline, a higher prevalence of cirrhosis, and a higher number of subjects previously treated with unsuccessful IFN-based regimens, probably due to a high rate of IFN-discontinuations. Moreover, a higher number of concomitant comorbidities (hypertension, diabetes, thyreopathies) was reported in patients with anxiety/depression, even if differences between the two groups were not statistically significant. Considered all these differences, it is interesting to highlight that, although group A patients had more hepatic and nonhepatic disabilities and a greater fragility, a lower SVR rate (79\%) was observed in psychotic patients compared to others (92\%) which was mostly due to a higher proportion of patients lost to follow-up. This could be expected considering some particular aspects characterizing psychotic disorders, probably not completely compensated by the antipsychotic treatment (disorganized behavior, impaired ability of sustained attention). In fact, when considering PP-analysis, a higher SVR-12 rate was observed $(97.9 \%$ in group A and $94.4 \%$ in group B), and our results were in line with recent studies $[13,15,16]$. Morevoer, in a subanalysis of study population the lack of achievement of SVR-12 was especially reported in former/active intravenous drug use. Finally, we cannot ruled out the possibility that, although patients received an appropriate treatment regimen, the highest proportion of $\mathrm{HCV}$ genotype 3 contributed to the lower response in group $\mathrm{B}$, in which was also described the unique relapse $(\mathrm{Y} 93 \mathrm{H}, \mathrm{NS} 5 \mathrm{~A}$ at treatment failure within 12 weeks after EOT).

Moreover, one of our main concerns before starting DAAs and during the course of treatment was the need of an adjustment or modification of the baseline psychiatric co-medication, which was prescribed in over 13.8\% of our patients, in agreement with psychiatrists. In fact, changes in psychiatric treatment before DAA-initiation seemed to play a role in treatment failure as it was associated with a higher number of lost to follow-up patients. The lack of a self-rated questionnaire evaluated after the completion of anti-HCV treatment failed to prove whether lost to follow-up patients was due to an underlying psychiatric disorder or other causes (poor 
medical awareness, doctor-patient relationship). Mostly, psychiatric drugs modifications were adopted among patients in treatment with antipsychotics (up to 19\% of cases); the majority of DAAs and antipsychotics shared a same major hepatic landmark in which both were CYP activators or substrates, leading to an increased/decreased exposure to the other drug. Fortunately, the wide availability of new DAA-regimens has nowadays reduced the need of mandatory therapeutic changes. Therefore, our study suggests that in these cases caution is warranted, considering the risk of altering a previous stable mental condition, and a long period of patient monitoring before starting DAAs is desirable. On the contrary, changes should be avoided if not strictly required.

Overall, DAA-based treatment was safe. Almost half of patients $(41.6 \%)$ experienced at least one mild-tomoderate adverse event. In general, no significant difference was observed in the occurrence and number of AEs between the two groups. As expected, RBV (used in comparable proportion between the two groups) was associated with more AEs in the course of treatment in comparison with patients treated with RBV-sparing regimens. Severe AEs occurred in only 10 patients (6.9\%). However, SAEs were less frequent than expected, probably due to the fact that the vast majority of patients had a compensated liver disease. Among 4 individuals who did not achieve EOT, 3/4 reported a treatment discontinuation due to SAEs, including seizure, intense headache, angina pectoris. Nevertheless, the few patients who experienced psychiatric symptoms such as depressive disorder and/or isolated generalized anxiety episode during DAA-treatment were exclusively among subjects in group A whereas none of the patients receiving antipsychotics showed the same disorders. Probably this interesting evidence might be explained by a major aptitude of these patients to perceive the initiation of a new treatment course as a stressing factor [27]. Usually, these symptoms were reported within the first month of treatment.

In a recent review published by Forton et al. [28] the HCV-clearance led to a valid improvement of neuropsychiatric manifestations. In disagreement with this study, in a recent Spanish study DAA-treatment had no impact on anxiety or depression during or after chronic hepatitis $\mathrm{C}$ infection treatment, even in high-risk patients with major psychiatric disorders [29]. We cannot confirm this findings as no posttreatment neurospychiatric evaluation was performed; however, in our experience, none of the psychiatric disorders reported during treatment had worsened at the end of treatment.

As widely demonstrated in literature, no difference in efficacy and safety rates were observed in HCV/HIV- coinfected patients compared to HCV-monoinfected. Even if the management of DDIs could be more complicated in these patients, for whom also antiretroviral therapy should be considered, changing ART before baseline did not affect therapy failure in our patients.

The main limitation of our study is its observational, retrospective nature; therefore, some data were not available for the totality of subjects; another limit is the restricted follow-up period after treatment and the lacking of a clinical follow-up based on questionnaires to assess self-reported outcomes.

\section{Conclusion}

Our study points out the complexity of the anti-HCV treatment of HCV-infected patients with psychiatric comorbidity and suggests that slightly lower SVR rates can be expected in psychotic patients while adverse events were more frequently reported among anxious/depressive patients. The study underlines as a careful evaluation of history and all possible drug-drug interactions before starting therapy can have a remarkable impact on the patient outcome, which was overall successful in our experience, thus encouraging a widespread use of DAAs also in such a "special population".

\section{Abbreviations \\ AEs: Adverse events; ART: Antiretroviral therapy; CNS: Central Nervous System; CTCAE: Common Terminology Criteria for Adverse Events; \\ CYP: Cytochrome; DAAs: Direct-acting Antiviral Agents; DCV: Daclatasvir; DDIs: Drug-drug interactions; DSV: Dasabuvir; EBR: Elbasvir; EMA: European Medicines Agency; EOT: End of treatment; FDA: Food and Drug Administration; GLE: Glecaprevir; GRZ: Grazoprevir; HCV: Hepatitis C virus; HIV: Human Immunodeficiency Virus; IDU: Injecting drug user; IFN: Interferon; IQR: Interquartile range; ITT: Intention-to-treat; LDV: Ledipasvir; MSM: Man who have sex with man; OBV: Ombitasvir; PIB: Pibrentasvir; PP: Per-protocol; PTV/r: Paritaprevir/ritonavir; RBV: Ribavirin; SAEs: Severe Adverse events; SD: Standard Deviation; SMV: Simeprevir; SOF: Sofosbuvir; SVR: Sustained Virological Response; VEL: Velpatasvir; VOX: Voxilaprevir}

\section{Acknowledgements}

Not applicable.

\section{Authors' contributions}

Conceptualization: NDG, LM, GA, AS. Methodology: NDG, LM, MM, AS Investigation and data curation: NDG, LD, MM. Formal analysis: NDG. Writing - original draft preparation: NDG. Writing - draft revision: LM, GA, AS. All authors have read and approved the manuscript.

Funding

No funding was obtained for this study.

\section{Availability of data and materials}

The dataset analyzed during the current study is available from the corresponding author on reasonable request.

\section{Ethics approval and consent to participate}

The study did not require approval from the ethics committee, according to the Italian law, since it was performed in the context of normal clinical routines (art.1, leg. Decree 211/2003). However, all patients referring to our institute provided a written consent at the time of first visit for the use of their data for research purposes. Patients were recruited retrospectively according to General Authorization to process personal data for research purposes (leg. Decree 9/ 2016). Data were previously anonymized, according to the requirements set by Italian Data protection Code (leg. Decree 196/2003). 


\section{Consent for publication}

Not applicable.

\section{Competing interests}

The authors declare that they have no competing interests.

Received: 12 September 2019 Accepted: 26 February 2020

Published online: 06 March 2020

\section{References}

1. WHO. GLOBAL HEPATITIS REPORT 2017. https://www.who.int/hepatitis/ publications/global-hepatitis-report2017/en/ Accessed 10 Aug 2019.

2. Revie D, Salahuddin SZ. Human cell types important for hepatitis $C$ virus replication in vivo and in vitro: old assertions and current evidence. Virol J. 2011 Jul 11:8:346,

3. Zignego AL, Craxi A. A Extrahepatic manifestations of hepatitis C virus infection. Clin Liver Dis. 2008;12:611-36.

4. Schaefer M, Capuron L, Friebe A, Diez-Quevedo C, Robaeys G, Neri S, et al. Hepatitis $C$ infection, antiviral treatment and mental health: a European expert consensus statement. J Hepatol. 2012;57:1379-90.

5. Martin LM, Sheridan MJ, Younossi ZM. The impact of liver disease on healthrelated quality of life: a review of the literature. Curr Gastroenterol Rep. 2002:4:79-83.

6. Yarlott L, Heald E, Forton D. Hepatitis C virus infection, and neurological and psychiatric disorders - a review. J Adv Res. 2017:8:139-48.

7. Adinolfi LE, Nevola R, Lus G, Restivo L, Guerrera B, Romano C, et al. Chronic hepatitis $C$ virus infection and neurological and psychiatric disorders: an overview. World J Gastroenterol. 2015;21:2269-80.

8. Lacey C, Ellen S, Devlin H, Wright E, Mijch A. Hepatitis C in psychiatry inpatients: testing rates, prevalence and risk behaviours. Australas Psychiatry. 2007;15:315-9.

9. Hosoda S, Takimura H, Shibayama M, Kanamura H, Ikeda K, Kumada H. Psychiatric symptoms related to interferon therapy for chronic hepatitis C: clinical features and prognosis. Psychiatry Clin Neurosci. 2000;54:565-72.

10. Niederau C, Mauss S, Schober A, Stoehr A, Zimmermann T, Waizmann M, et al. Predictive factors for sustained virological response after treatment with pegylated interferon a-2a and ribavirin in patients infected with HCV genotypes 2 and 3. PLoS One. 2014;9:e107592.

11. European Association for the Study of the Liver. EASL recommendations on treatment of hepatitis C 2018. J Hepatol. 2018:69:461-511.

12. Jain MK, Thamer M, Therapondos G, Shiffman ML, Kshirsagar O, Clark C. Has access to hepatitis $C$ virus therapy changed for patients with mental health or substance use disorders in the direct-acting-antiviral period? Hepatology. 2019;69:51-63.

13. Back D, Belperio P, Bondin M, Negro F, Talal AH, Park C, et al. Efficacy and safety of glecaprevir/pibrentasvir in patients with chronic HCV infection and psychiatric disorders: an integrated analysis. J Viral Hepat. 2019;26:951-60.

14. Christensen S, Buggisch P, Mauss S, Böker K, Schott E, Klinker H, et al. Directacting antiviral treatment of chronic HCV-infected patients on opioid substitution therapy: still a concern in clinical practice? Addiction. 2018; 113(5):868-82

15. Miarons M, Sánchez-Ulayar A, Sempere G, Marín S, Castellví JM. New directacting antivirals for hepatitis $C$ treatment and neuropsychiatric symptoms in psychiatric risk groups. Eur J Hosp Pharm. 2018;0:1-6.

16. Gayam V, Jegede O, Tiongson B, Mandal AK, Sidhu J, Garlapati P. Outcomes of direct-acting antiviral treatment of psychiatric patients with comorbid hepatitis C virus infection. Dig Dis. 2019;6:1-8.

17. EASL. Recommendations on Treatment of Hepatitis C 2015-2018. https:// easl.eu/publications/clinical-practice-guidelines Accessed 11 Aug 2019

18. Lazarus JV, Wictor SZ, Colombo M, Thursz M, EASL International Liver Foundation. Micro-elimination - a path to global elimination of hepatitis $C$. J Hepatol. 2017;67:665-6.

19. Larrey D, Ripault MP, Pageaux GP. Patient adherence issues in the treatment of hepatitis C. Patient Prefer Adherence. 2014;8:763-73.

20. Dirks $M$, Pflugrad H, Haag K. Persistent neuropsychiatric impairment in HCV patients despite clearance of the virus? J Viral Hepat. 2017;24:541-50.

21. Yovtcheva SP, Rifai MA, Moles JK, Van der Linden BJ. Psychiatric comorbidity among hepatitis C-positive patients. Psychosomatics. 2001;42:411-5.

22. El Serag HB, Kunik M, Richardson P, Rabeneck L. Psychiatric disorders among veterans with hepatitis C infection. Gastroenterology. 2002;123:476-82.
23. Carta MG, Angst J, Moro MF, Mura G, Hardoy MC, Balestrieri C, et al. Association of chronic hepatitis $\mathrm{C}$ with recurrent brief depression. J Affect Disord. 2012;141:361-6.

24. Wu JY, Shabolt B, Teoh N, Blunn A, To C, Rodriguez-Morales I, et al. Influence of psychiatric diagnosis on treatment uptake and interferon side effects in patients with hepatitis C. J Gastroenterol Hepatol. 2014;29:1258-64.

25. Sundberg I, Lannergård A, Ramklint M, Cunningham JL. Direct-acting antiviral treatment in real world patients with hepatitis $C$ not associated with psychiatric side effects: a prospective observational study. BMC Psychiatry. 2018;18:157.

26. Bruno G, Saracino A, Scudeller L, Fabrizio C, Dell'Acqua R, Milano E, et al. HCV mono-infected and HIV/HCV co-infected individuals treated with direct-acting antivirals: to what extent do they differ? Int J Infect Dis. 2017;62:64-71.

27. Quato DM, Ozenberger K, Olfson M. Prevalence of prescription medications with depression as a potential adverse effect among adults in the United States. JAMA. 2018;319:2289-98.

28. Forton $D$, Weissenborn $K$, Bondin M, Cacoub P. Expert opinion on managing chronic HCV in patients with neuropsychiatric manifestations. Antivir Ther. 2018;23:47-55.

29. Gallach M, Vergara M, da Costa JP, Miquel M, Casas M, Sanchez-Delgado J, et al. Impact of treatment with direct-acting antivirals on anxiety and depression in chronic hepatitis. PLoS ONE. 2018;13:e0208112.

\section{Publisher's Note}

Springer Nature remains neutral with regard to jurisdictional claims in published maps and institutional affiliations.
Ready to submit your research? Choose BMC and benefit from:

- fast, convenient online submission

- thorough peer review by experienced researchers in your field

- rapid publication on acceptance

- support for research data, including large and complex data types

- gold Open Access which fosters wider collaboration and increased citations

- maximum visibility for your research: over $100 \mathrm{M}$ website views per year

At $\mathrm{BMC}$, research is always in progress.

Learn more biomedcentral.com/submissions 